\title{
Influência dos distúrbios respiratórios no desenvolvimento das maloclusões
}

\section{Influence of respiratory disorders in the development of maloclusions}

\author{
Gabriela de Albuquerque Almeida Figueredo ${ }^{1}$ \\ Diana Maria Arraes Feitosa ${ }^{2}$ \\ Ginna Kércia Matos Gonçalves ${ }^{3}$ \\ Karla Shangela da Silva Alves ${ }^{4}$
}

\begin{abstract}
RESUMO
Objetivo: Investigar a relação entre maloclusões e desordens de ordem respiratória: rinite alérgica, hipertrofia de adenoide e de tonsilas palatinas em crianças.

Material e Métodos: Realizou-se levantamento bibliográfico sobre o tema na base de dados PubMed, de 2007 a 2017, buscando artigos em inglês e português com as palavras-chave: maloclusão, odontopediatria, respiração bucal, adenoide, mordida cruzada, rinite alérgica, amídalas, em português e inglês. Foram incluídas as bibliografias que contivessem os descritores e, de exclusão, os que não atendessem à temática ou que não estivessem disponíveis na versão completa. Foi feita uma revisão da literatura, desenvolvida seguindo os preceitos de um estudo explanatório, por meio de pesquisa bibliográfica dividida em etapas. Muitos estudos mostraram que existe relação entre as desordens do trato respiratório superior (rinite alérgica, hipertrofia de adenoide e de amídalas) e alguns tipos de maloclusão.

Conclusão: Ancorado nos achados bibliográficos, existe uma relação das desordens do trato respiratório e as maloclusões, por isso um acompanhamento multiprofissional é imprescindível, apontando relação direta entre prognóstico e precocidade no início do tratamento.
\end{abstract}

Palavras-chave: Doenças Respiratórias. Má Oclusão. Respiração Bucal. Rinite Alérgica. Tonsila Faríngea.

\begin{abstract}
Objective: The aim of this study was to investigate the relationship between malocclusion and respiratory disorders: allergic rhinitis, adenoid hypertrophy and palatine tonsils in children.

Material and Methods: A bibliographic survey was performed on the subject in the PubMed database, from 2007 to 2017, searching for articles with the following keywords: malocclusion, pediatric dentistry, mouth breathing, adenoid, crossbite, allergic rhinitis, tonsils, in both Portuguese and English. The bibliographies containing the descriptors and of exclusion were included that did not attend the theme or that were not available in the complete version. A review of the literature, developed following the precepts of an explanatory study, was made through a bibliographical research divided into stages. Many studies have shown that there is a relationship between upper respiratory tract disorders (allergic rhinitis, adenoid and tonsil hypertrophy) and some types of malocclusion.

Conclusion: Based on the bibliographical findings show a relationship of respiratory tract disorders and malocclusions, so a multiprofessional follow-up is essential, pointing out a direct relationship between prognosis and precocity at the beginning of treatment.
\end{abstract}

Keywords: Respiratory Tract Diseases. Malocclusion. Mouth Breathing. Rhinitis, Allergic. Adenoids.

\footnotetext{
Especialista em Odontopediatria pela Academia Cearense de Odontologia. Fortaleza, CE, Brasil.

Especialista em Odontopediatria pela Associação Brasileira de Odontologia. Fortaleza, CE, Brasil.

Mestre em Odontopediatria pela Universidade Cruzeiro do Sul. São Paulo, SP. Brasil.

Professora Auxiliar da Disciplina de Odontopediatria da Universidade de Fortaleza. Fortaleza, CE, Brasil.
} 


\section{INTRODUÇÃO}

Maloclusão é uma condição de desvio da normalidade, na forma como maxila e mandíbula, com suas respectivas arcadas dentárias, se relacionam. ${ }^{(1)}$ Pode ser desencadeada por uma série de fatores como: cárie dentária, fatores genéticos, hábitos de sucção não-nutritiva que se estabelecem por longo período, rotinas de alimentação incorreta (com pouco uso da mastigação), posicionamento e função anormais da língua e postura corporal. ${ }^{1,2,3}$

Já os distúrbios respiratórios são condições patológicas que afetam o sistema respiratório e o seu correto funcionamento. Podem influenciar no crescimento e desenvolvimento das estruturas craniofaciais e, por conseguinte, na oclusão dental. ${ }^{(4)} \mathrm{A}$ literatura aponta que alguns desses distúrbios comprometem a respiração nasal exclusiva, levando seus portadores a respirarem também pela boca e a desencadear maloclusões. ${ }^{5,6,7}$

A respiração exclusiva pelo nariz promove o correto posicionamento das estruturas fonoarticulatórias e protege as vias aéreas, além de proporcionar o bom funcionamento de todo o complexo estomatognático. ${ }^{8}$ Os distúrbios respiratórios do trato superior são, de acordo com a literatura, os que mais levam ao desenvolvimento da respiração bucal. ${ }^{4,9,10} \mathrm{Em}$ consequência à respiração bucal, pode-se prever o surgimento das maloclusões. ${ }^{2,4}$

Nesse sentido, há um considerável número de alterações vistas como consequências dos distúrbios respiratórios, como deformidades faciais, desordens esqueléticas, postura incorreta da língua na cavidade oral, alterações no posicionamento dos dentes, além de gengivite, xerostomia e outros. ${ }^{5,8,10,11}$

No caso do comprometimento da respiração pelo nariz, há uma adaptação do organismo para sanar tal comprometimento, de forma a se conseguir suprir a quantidade de ar que se necessita respirar, o que leva a uma alteração postural ${ }^{7,12}$ e à instalação da respiração bucal. ${ }^{3}$

De acordo com os estudos, $, 5,6,7,12,13$ as obstruções que mais levam à respiração bucal e, por conseguinte, potencializam as chances de desenvolver problemas de maloclusão são a rinite alérgica e a hipertrofia de tonsilas.

Rinite alérgica é citada como uma das disfunções crônicas que mais afetam crianças, ${ }^{14}$ comprometendo a mucosa nasal. Afirma-se que um dos achados do exame físico sugestivo de rinite alérgica é a respiração bucal, e uma das suas complicações é o agravamento da hipertrofia de adenoide. ${ }^{15}$

A hipertrofia das tonsilas leva à obstrução das vias aéreas superiores, estabelecendo-se um quadro de obstrução nasal, respiração bucal de suplência e roncos noturnos, queixas de disfagia, problemas olfatórios e de paladar e alterações da voz, como hiponasalidade. ${ }^{15}$

A face adenoideana é caracterizada, segundo Linder-Aronson, ${ }^{16}$ por um selamento labial incompetente, um arco dentário superior estreito, incisivos inferiores lingualizados, dentes incisivos proeminentes, dentes agrupados, aumento da altura da face anterior, entre outras características - como olheiras - em comparação com faces de crianças sem hipertrofia de adenoide (tonsilas faríngeas).

A hipertrofia das amídalas (tonsilas palatinas) pode afetar o desenvolvimento craniofacial de crianças, nas quais mandíbula e língua deslocam-se para baixo e para trás, ocasionando alterações posturais que prejudicam o equilíbrio normal entre as forças exercidas nos dentes e tecidos moles da face pela língua e pelo lábio, o que gera alterações maxilofaciais. Surgem daí as maloclusões dentárias, classificadas em mordida aberta, mordida cruzada e protrusão maxilar. ${ }^{15}$

Em contrapartida, são poucas as referências encontradas na literatura que mostram a relação entre maloclusões e problemas de obstrução respiratória, especificamente a hi- 
pertrofia de adenoide e/ou de tonsilas palatinas e a rinite alérgica em crianças; devido a isso, considera-se esta revisão de literatura de grande importância, pois por meio desta buscou-se confirmar a correlação entre má oclusão e as condições supracitadas.

O objetivo deste trabalho foi investigar a relação entre maloclusões e desordens de ordem respiratória: rinite alérgica, hipertrofia de tonsilas faríngeas e palatinas em crianças, tentando associar o desenvolvimento da respiração bucal como consequência dessas condições.

\section{Metodologia}

Trata-se de uma revisão de literatura, desenvolvida seguindo os preceitos de uma leitura exploratória, ${ }^{17}$ por meio de pesquisa bibliográfica dividida em etapas: seleção das fontes, coleta de dados, análise e interpretação e discussão dos resultados.

Foram utilizados três livros, divididos em Otorrinolaringologia, Ortodontia, Odontopediatria, no idioma português; artigos científicos sobre a temática, acessados nas bases de dados SCIELO, MEDLINE, PUBMED, LILACS, publicados nos últimos 10 anos, sendo seis artigos nacionais, 10 internacionais, disponíveis completos e on-line.

Os seguintes descritores foram utilizados: "malocclusion", "tongue", "pediatric dentistry", "mouth breathing", "adenoids", "crossbite", "allergic rhinitis", também em português. Para a seleção das fontes, foram consideradas como critério de inclusão as bibliografias que abordassem os descritores e foram excluídos aqueles que não atendessem à temática ou que não estivessem disponíveis na versão completa.

A coleta de dados consistiu em leitura explanatória de todo o material selecionado; leitura seletiva; registro das informações extraídas das fontes em instrumento específico (autores, ano, resultados e conclusões).

$\mathrm{Na}$ etapa de análise e interpretação dos resultados, foi realizada uma leitura analítica com a finalidade de ordenar e sumariar as informações contidas nas fontes, de forma que estas possibilitassem a obtenção de respostas ao problema de pesquisa.

$\mathrm{Na}$ discussão dos resultados, categorias que emergiam da etapa anterior, foram analisadas e discutidas a partir do referencial teórico relativo ao estudo.

\section{Resultados}

De acordo com os artigos estudados, a integridade das estruturas que compõem o sistema respiratório é fundamental para permitir uma respiração eficiente. ${ }^{(18)}$ Durante a respiração nasal, é necessário que a boca se feche em algum ponto. Normalmente, isso ocorre anteriormente, pelo selamento dos lábios.

A ausência da respiração nasal exclusiva impede a efetiva reabsorção óssea no interior das fossas nasais e a deposição óssea na parte externa, ${ }^{19}$ promovendo aposição óssea na região acima do palato e exercendo efeito prejudicial sobre o crescimento e o desenvolvimento faciais normais, ocorrendo, inclusive, desequilíbrios entre os vários componentes morfofuncionais da face, os quais serão refletidos em alterações funcionais. ${ }^{20}$ Quando um indivíduo apresenta essa condição por, pelo menos, seis meses, já pode ser considerado um respirador bucal. ${ }^{21}$

Desse modo, o sistema respiratório superior, quando apresenta alguma desordem, pode provocar problemas não somente no crescimento e desenvolvimento da estrutura craniofacial, como também, por conseguinte, dificuldades na oclusão dentária, favorecendo o surgimento das maloclusões. Sabe-se que a obstrução de vias aéreas superiores interfere na pressão inspiratória, uma vez que o baixo fluxo de ar pelas narinas e a ausência da pressão da língua contra o palato induzem à hipoplasia do seio maxilar, ao estreitamento das cavidades nasais e do arco dental superior, o que favorece a maloclusão dentária. ${ }^{4}$ 
De acordo com a literatura, as principais maloclusões associadas aos distúrbios respiratórios de ordem obstrutiva são a mordida aberta anterior e a mordida cruzada posterior. O tratamento desses casos geralmente exige uma abordagem multiprofissional, o que envolve cirurgião-dentista (capacitado a realizar uma conduta de ordem ortopédica e ortodôntica preventiva), otorrinolaringologista (para tratar o problema obstrutivo) e fonoaudiólogo (que atua na correção da respiração bucal, entre outras desordens atreladas ao problema em questão). O quadro 1, a seguir, mostra um resumo dos trabalhos estudados.

Quadro 1 - Descrição da relação entre distúrbios respiratórios e maloclusões, de acordo com o ano de publicação.

\begin{tabular}{|c|c|c|c|c|}
\hline Ano & $\begin{array}{l}\text { Há relação entre } \\
\text { distúrbios respirató- } \\
\text { rios e maloclusões }\end{array}$ & $\begin{array}{l}\text { Distúrbio } \\
\text { respiratório } \\
\text { citado }\end{array}$ & $\begin{array}{l}\text { Perfil facial detec- } \\
\text { tado em crianças } \\
\text { com distúrbios } \\
\text { respiratórios }\end{array}$ & Maloclusões citadas \\
\hline $\begin{array}{l}\text { Fields et al., } \\
1991^{26}\end{array}$ & Sim & - & $\begin{array}{l}\text { Aumento da altura } \\
\text { facial }\end{array}$ & $\begin{array}{l}\text { Mordida aberta anterior, } \\
\text { aumento do overjet, estrei- } \\
\text { tamento do palato }\end{array}$ \\
\hline $\begin{array}{l}\text { Souki et al., } \\
2009^{9}\end{array}$ & Sim & - & - & $\begin{array}{l}\text { Mordida cruzada posterior, } \\
\text { mordida aberta anterior, } \\
\text { maloclusão de Classe II }\end{array}$ \\
\hline $\begin{array}{l}\text { Hopatiene et al., } \\
2013^{3}\end{array}$ & Não & $\begin{array}{l}\text { Não focou em } \\
\text { distúrbios respi- } \\
\text { ratórios }\end{array}$ & - & $\begin{array}{l}\text { Graus de apinhamento, } \\
\text { mordida cruzada posterior }\end{array}$ \\
\hline $\begin{array}{l}\text { Luzzi et al., } \\
2013^{12}\end{array}$ & Sim & Rinite Alérgica & - & $\begin{array}{l}\text { Mordida cruzada posterior } \\
\text { e aumento do overjet }\end{array}$ \\
\hline AAPD, $2014^{25}$ & Sim & - & $\begin{array}{l}\text { Aumento da altura } \\
\text { facial }\end{array}$ & $\begin{array}{l}\text { Mordida aberta anterior, } \\
\text { aumento do overjet, estrei- } \\
\text { tamento do palato }\end{array}$ \\
\hline $\begin{array}{l}\text { Basheer et al., } \\
2014^{5}\end{array}$ & Sim & $\begin{array}{l}\text { Hipertrofia de } \\
\text { adenoide }\end{array}$ & $\begin{array}{l}\text { Perfil facial convexo } \\
\text { e profundidade do } \\
\text { sulco mentolabial } \\
\text { acentuados }\end{array}$ & $\begin{array}{c}\text { Proclinação de incisivos } \\
\text { inferiores }\end{array}$ \\
\hline $\begin{array}{l}\text { Agostinho et al., } \\
2015^{14}\end{array}$ & Sim & Rinite Alérgica & Faces mais longas & $\begin{array}{l}\text { Maxilas e mandíbulas mais } \\
\text { curtas, mordida aberta }\end{array}$ \\
\hline $\begin{array}{l}\text { Feres et al., } \\
2015^{23}\end{array}$ & - & $\begin{array}{l}\text { Hipertrofia de } \\
\text { adenoide }\end{array}$ & $\begin{array}{c}\text { Tendência ao cres- } \\
\text { cimento vertical } \\
\text { acentuado, perfil } \\
\text { convexo, retrusão } \\
\text { mandibular }\end{array}$ & Não citou \\
\hline $\begin{array}{l}\text { Majorona et al., } \\
2015^{24}\end{array}$ & Sim & - & $\begin{array}{l}\text { Altura facial aumen- } \\
\text { tada }\end{array}$ & $\begin{array}{l}\text { Mordida aberta anterior, } \\
\text { aumento do overjet, palato } \\
\text { estreito }\end{array}$ \\
\hline $\begin{array}{l}\text { Gomes et al., } \\
2016^{6}\end{array}$ & Sim & $\begin{array}{l}\text { Anormalidades } \\
\text { de orofaringe }\end{array}$ & $\begin{array}{l}\text { Aumento da altura } \\
\text { facial }\end{array}$ & $\begin{array}{l}\text { Mordida aberta anterior, } \\
\text { mordida cruzada posterior }\end{array}$ \\
\hline $\begin{array}{c}\text { Imband et al., } \\
2016^{4}\end{array}$ & Sim & Rinite Alérgica & Dolicofacial & Não citou \\
\hline $\begin{array}{l}\text { Zhu et al., } \\
2016^{13}\end{array}$ & Sinaliza que sim & $\begin{array}{l}\text { Hipertrofia de } \\
\text { adenoide e de } \\
\text { amídalas }\end{array}$ & - & $\begin{array}{l}\text { Arco dental maxilar poste- } \\
\text { rior mais estreito, overjet, } \\
\text { overbite }\end{array}$ \\
\hline $\begin{array}{l}\text { Milanesi et al., } \\
2017^{22}\end{array}$ & Sim & Rinite Alérgica & - & $\begin{array}{l}\text { Redução da largura do } \\
\text { palato duro }\end{array}$ \\
\hline
\end{tabular}




\section{Dıscussão}

O presente estudo mostrou que, de acordo com os relatos, as causas mais frequentes da respiração bucal de suplência são obstruções nasais e/ou obstruções faríngeas. A ausência da respiração nasal exclusiva ocasiona danos ao ser humano. ${ }^{27}$ Os prejuízos da respiração bucal são vários, e entre eles são citadas as maloclusões em diversos estudos. 3,4,6,9,12-14,24

Nesse sentido, nota-se que há uma relação estatisticamente significativa entre rinite alérgica e maloclusão. ${ }^{12,14}$ Crianças com reação positiva a aeroalérgenos, respiração bucal e má-oclusão dentária têm faces mais longas, maxilas e mandíbulas mais curtas, tendência à mordida aberta e ao espaço reduzido de vias aéreas (provavelmente devido a uma posição mais anterior da língua), quando comparadas às crianças respiradoras nasais. ${ }^{14}$

Percebe-se uma convergência entre os estudos na associação entre padrão de crescimento dolicofacial e respiradores bucais, mas Imband et al. (2016) afirmam que, em pacientes com rinite alérgica, independente da presença de respiração bucal, a tendência do crescimento dolicofacial não é observada. Existe uma tendência maior de rinite em crianças com maloclusão, comparado à população geral, que é cerca de $30 \%$. ${ }^{(4)}$

Além disso, Milanesi et al. (2017) ${ }^{2}$ compararam a permeabilidade nasal e características otorrinolaringológicas e orofaciais em 123 crianças de 6 a 12 anos de idade, detectando que a permeabilidade nasal foi menor em crianças com sono agitado, sinais e sintomas de rinite, redução da largura do palato duro e alterações na mastigação, deglutição e funções de fala. $O$ trabalho enfatizou também que a maioria das crianças apresentava sinais e sintomas de rinite alérgica. ${ }^{(2)}$

A presença de mordida cruzada posterior é superior em crianças respiradoras bucais do que na população em geral, ${ }^{6,9}$ e também existe maior probabilidade de haver mordida aberta anterior e maloclusão de Classe II nas dentições mista e permanente de respiradores bucais. Contudo, Souki et al. (2009) afirmaram que o tamanho da tonsila faríngea e/ ou das tonsilas palatinas e a prevalência de rinite alérgica não eram fatores de risco para o desenvolvimento de maloclusão de Classe II, mordida aberta anterior ou cruzada posterior, Gomes et al. (2016) observaram associações significativas entre mordida aberta anterior e mordida cruzada posterior e as anormalidades de oronasofaringe em pré-escolares. Muitos estudos reforçam os resultados, pois apontam que, quando há prejuízo da respiração nasal, há uma contribuição para o aumento da altura facial, o desenvolvimento de mordida aberta anterior, o crescimento de overjet e o estreitamento do palato. ${ }^{24,25,26}$

Feres et al. (2015) compararam o padrão cefalométrico de crianças com e sem obstrução de vias aéreas devido à hipertrofia de adenoide. Os resultados indicaram que esses pacientes demonstraram tendência ao crescimento vertical acentuado, ao perfil convexo e à retrusão mandibular; contudo, não houve diferenças entre pacientes com e sem obstrução, em relação às variáveis cefalométricas.

Ademais, num estudo realizado com 50 crianças de idade entre 6 e 12 anos, respiradoras bucais, com e sem hipertrofia de adenoide, objetivou-se analisar desordens de natureza dentária e de tecidos moles. Foi concluído que todos os pacientes apresentavam proclinação dos incisivos inferiores, incompetência no selamento labial e perfil facial convexo. ${ }^{5}$

Já o estudo interdisciplinar ${ }^{3}$ para avaliar a correlação entre maloclusão e a postura corporal mostrou que não existe relação entre grau de apinhamento, presença de mordida cruzada posterior, parâmetros ortopédicos e um padrão de respiração, contradizendo a maioria dos estudos publicados, apesar de levar em consideração apenas a relação postura 
corporal e maloclusão, e não focar somente nas obstruções de ordem respiratória.

O abrangente estudo ${ }^{13}$ consistiu numa meta-análise e revisão sistemática com o objetivo de determinar o efeito das cirurgias de remoção de adenoide (adenoidectomia) e de amídalas (tonsilectomia/amidalectomia) no crescimento da morfologia dental em crianças com obstrução de vias aéreas. Foi detectado que crianças com obstrução das vias respiratórias tiveram um arco dental maxilar posterior mais estreito em relação a crianças sem obstrução; além disso, a adenoidectomia e tonsilectomia promoveram uma alteração significativa na respiração nasal em crianças com obstrução, com uma evidente tendência à normalização, em termos de largura do arco dentário, maloclusão, altura do palato, overjet e overbite, perímetro e comprimento do arco dentário. Porém, não houve diferenças significativas evidentes entre o grupo que se submeteu às cirurgias e o grupo normal. Os autores alegam limitações para a realização do trabalho em questão por falta de ensaios controlados e reduzido número de estudos acerca do assunto.

\section{Considerações finAIS}

De acordo com a revisão da literatura, tornou-se possível verificar que existe relação entre as desordens do trato respiratório superior (rinite alérgica, hipertrofia de adenoide e de amídalas) e alguns tipos de maloclusão. A respiração bucal inicia-se como um complemento ou mesmo um substituto à respiração nasal insuficiente, que pode ser causada por essas desordens. Nesse sentido, acompanhando a abertura da boca na necessidade de respirar, a língua interpõe-se, e a consequência disso é o estabelecimento de um desequilíbrio das pressões que, quando juntas e equilibradas, contribuem para um desenvolvimento estomatognático harmônico. Diante disso, desenvolve-se um desequilíbrio na oclusão e na harmonia da face.

Nesse contexto, as desordens respiratórias de trato superior - seja a rinite alérgica, seja a hipertrofia de adenoide e de amídalas - têm papel influenciador nas maloclusões dentárias. Assim, um acompanhamento multiprofissional é imprescindível, incluindo pediatra, otorrinolaringologista, fonoaudiólogo, odontopediatra e ortodontista; pois, o quanto antes acontecerem a abordagem e a intervenção, tanto melhor o prognóstico.

Este trabalho mostrou a necessidade de mais estudos com o intuito tanto de informar profissionais e leigos na área, quanto de fortalecer um consenso entre autores e estudiosos do assunto.

\section{CONFLITOS DE INTERESSES}

Os autores declaram que não há conflitos de interesses.

FONTES DE FINANCIAMENTO Sem financiamento.

\section{REFERÊNCIAS}

1. Corrêa MSNP. Odontopediatria na primeira infância, 3. ed. São Paulo (SP): Santos; 2011, p. 135-140.

2. Gracco A., Perri A., Siviero L., Alessandri F., Cocilovo F., Stellini E. Multidisciplinary correction of anterior open bite relapse and upper airway obstruction. Korean J Orthod. 2015; 45 (1): 47 56. http://dx.doi.org/10.4041/kjod.2015.45.1.47

3. Lopatiene K., Smailiené D., Sidlauskiené M., Cekanauskas E., Valaikaité R., Pribuišienè R. An interdisciplinary study of orthodontic, orthopedic, and otorhinolaryngological findings in 12-14-year-old preorthodontic children. Medicina (Kaunas) 2013; 49 (11): 479-86. https://www.ncbi.nlm.nih.gov/ pubmed/24823929

4. Imbaud TCS, Mallozi MC, Domingos VBTC, Solé D. Frequency of rhinitis and orofacial disorders in patients with dental malocclusion. Rev paul pediatr. 2016; 34 (2): 184-88. http://dx.doi. org/10.1016/j.rppede.2016.02.009 
5. Basheer B., Hegde KS, Bhat SS, Umar D., Baroudi $\mathrm{K}$. Influence of mouth breathing on the dentofacial growth of children: a cephalometric study. J Int Oral Health. 2014; 6 (6): 505. https://www.ncbi.nlm.nih.gov/pmc/articles/ PMC4295456/

6. Gomes GB, Vieira-Andrade RG, Sousa RV, Firmino RT, Paiva SM, Marques LS, et al. Association between oronasopharyngeal abnormalities and malocclusion in Northeastern Brazilian preschoolers. Dental Press J. Orthod. 2016; 21 (3): 39-45. http://dx.doi.org/10.1590/21776709.21.3.039-045.oar.

7. Šidlauskienè M., Smailienè D., Lopatienè K., Čekanauskas E., Pribuišienè R., Šidlauskas M. Relationships between Malocclusion, Body Posture, and Nasopharyngeal Pathology in Pre-Orthodontic Children. Med Sci Monit 2015; 21: 1.765-73. http://dx.doi.org/10.12659/ MSM.893395

8. Hitosa SF, Arakaki R., Solé D., Weckx LL. Oral breathing and speech disorders in children. J. Pediatr. (Rio J.) 2013; 89 (4): 361-65. https:// doi.org/10.1016/j.jped.2012.12.007

9. Souki $B Q$, Pimenta GB, Souki MQ, Franco LP, Becker HM, Pinto JA. Prevalence of malocclusion among mouth breathing children: Do expectations meet reality? Int $\mathbf{J}$ Pediatr Otorhinolaryngol. 2009; 73 (5): 767-73. https://doi. org/10.1016/j.ijporl.2009.02.006

10. Pacheco MC, Casagrande CF, Teixeira LP, Finck NS, de Araújo MT. Guidelines proposal for clinical recognition of mouth breathing children. Dental Press J. Orthod. 2015; 20 (4): 39-44. http://dx.doi.org/10.1590/2176-9451.20.4.039044.oar

11. Peltomäki, T. The effect of mode of breathing on craniofacial growth - Revisited. Eur J Orthod. 2007; 29 (5): 426-29. https://doi.org/10.1093/ ejo/cjm055

12. Luzzi V., Lerardo G., Viscogliosi A., Fabbrizi M., Consoli G., Vozza I, et al. Allergic rhinitis as a possible risk factor for malocclusion: A casecontrol study in children. Int J Paediatr Dent. 2013; 23 (4): 274-78. https://doi.org/10.1111/ ipd.12003

13. Zhu Y., Li J., Tang Y., Wang X., Xue X., Sun $H_{\text {., }}$ et al. Dental arch dimensional changes after adenoidectomy or tonsillectomy in children with airway obstruction: a metaanalysis and systematic review under PRISMA guidelines. Medicine (Baltimore) 2016; 95 (39): e 4976. http://dx.doi.org/10.1097/ MD.0000000000004976

14. Agostinho HA, Furtado IÃ, Silva FS, Ustrell Torrent J. Cephalometric Evaluation of Children with Allergic Rhinitis and Mouth Breathing. Acta Med Port. 2015; 28 (3): 316-21. https://www. ncbi.nlm.nih.gov/pubmed/26421783

15. Di Francesco RC, Bento RF. Repercussões da obstrução nasal no crescimento craniofacia. In: Di Francesco RC, Bento RF. Otorrinolaringologia na Infância, 2. ed. Barueri: São Paulo; 2012, p. 96.

16. Linder-Aronson S. Adenoids. Their effects on mode of breathing and nasal airflow and their relationship to characteristics of the facial skeleton and the dentition. A biometric, rhino-manometric and cephalometro-radiographic study on children with and without adenoids. Acta Otolaryngol Suppl. 1970; 265: 1-132. https://www. ncbi.nlm.nih.gov/pubmed/5272140

17. Gil AC. Como elaborar projetos de pesquisa, 4. ed. São Paulo: Atlas S.A; 2002, p. 77-8.

18. Ramires RR, Ferreira LP, Marchesan IQ, Cattoni DM, Silva MA. Proposal for facial type determination based on anthropometry. J Soc Bras Fonoaudiol. 2011; 23 (3): 195-200. https:// www.ncbi.nlm.nih.gov/pubmed/22012152

19. Mezzomo CL, Machado PG, Pacheco AB, Gonçalves BFT, Hoffmann CF. As implicações da classe II de angle e da desproporção esquelética tipo classe II no aspecto miofuncional. Rev. CEFAC 2010; 13 (4): 728-34. http://dx.doi. org/10.1590/S1516-18462010005000079

20. Sannomiya EK, Bommarito S., Calles A. Avaliação do tamanho da adenóide por meio da radiografia cefalométrica em norma lateral em indivíduos com má oclusão de classe I, II e III. Ciênc Odonto Bras. 2005; 8 (3): 46-54, jul./set. 2005. http://dx.doi.org/10.14295/bds.2005.v8i3.429

21. Rizzo MC. O respirador bucal. In: Naspitz CK. Alergias respiratórias. São Paulo: Vivali; 2003, p. 98-110.

22. Milanesi JM, Berwig LC, Schuch LH, Ritzel RA, Silva AMTD, Corrêa ECR. Nasal patency and 
otorhinolaryngologic-orofacial features in children. Braz J Otorhinolaryngol. 2017; S18088694(17)30195-7. https://doi.org/10.1016/j. bjorl.2017.10.014

23. Feres MFN, Muniz TS, Andrade SH, Lemos MM, Pignatari SSN. Craniofacial skeletal pattern: is it really correlated with the degree of adenoid obstruction? Dental Press J. Orthod. 2015; 20 (4): 68-75. http://dx.doi.org/10.1590/21769451.20.4.068-075.oar

24. Majorana A., Bardellini E., Amadori F., Conti G., Polimeni A. Timetable for oral prevention in childhood-developing dentition and oral habits: a current opinion. Prog Orthod. 2015; 16: 39. https://doi.org/10.1186/s40510-015-0107-8
25. American Academy of Pediatric Dentistry. Guideline on management of the developing dentition and occlusion in pediatric dentistry. Clinical Practice Guidelines. 2014; 37 (6): 253-65.

http://www.aapd.org/media/Policies_Guidelines/G_ DevelopDentition.pdf

26. Fields HW, Warren DW, Black K., Phillips CL. Relationship between vertical dentofacial morphology and respiration in adolescents. Am J Orthod Dentofacial Othop. 1991; 99 (2): 147-54. https://www.ncbi.nlm.nih.gov/pubmed/1990824

27. Marchesan IQ. Fundamentos em fonoaudiologia: Aspectos Clínicos da Motricidade Oral. Nova Guanabara; 1998, p. 160.

Submetido em: 2-10-2018

Aceito em: 8-4-2019 